\title{
Author Correction: Numerical Analysis of Railways on Soft Soil Under Various Train Speeds
}

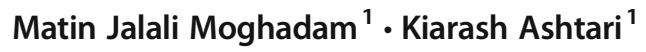

Published online: 16 December 2019

(C) Springer Science+Business Media, LLC, part of Springer Nature 2019

\section{Author Correction: Transportation Infrastructure Geotechnology https://doi.org/10.1007/s40515-019-00092-9}

The original version of this article unfortunately contained a mistake. The caption of figures 13 and 14 were incorrect. The correct captions are provided below:

Fig. 13 Lateral deformation of the embankment before passing the train

Fig. 14 Lateral deformation of the embankment under passing the train

The authors' apologizes for the oversight and for any confusion it may have caused.

The original article has been corrected.

Matin Jalali Moghadam

Kiarash Ashtari
ashtari.kiarash@gmail.com

1 Tehran, Iran

记 Springer 